\title{
Quantitative Compositional Mapping of Particles from the Apollo 17 Core 73002
}

Sarah Valencia ${ }^{1}$, Emma Bullock ${ }^{2}$, Corrigan Cari $^{3}$, Natalie Curran ${ }^{4}$, Barbara Cohen ${ }^{5}$ and ANGSA Science Team $^{6}$

${ }^{1}$ University of Maryland College Park, United States, ${ }^{2}$ Carnegie Institution for Science, WASHINGTON, District of Columbia, United States, ${ }^{3}$ Smithsonian Museum of Natural History, United States, ${ }^{4}$ Catholic University of America, United States, ${ }^{5}$ NASA Goddard Spaceflight Center, United States, ${ }^{6}$ NASA, United States

During the Apollo 17 mission in 1972, a $70 \mathrm{~cm}$ double-drive tube core sample was taken and sealed on the lunar surface as a lower drive tube (73001) and an upper drive tube (73002). For the benefit of future scientific investigations, NASA had the intention of preserving the samples for methods and technologies not yet available to the scientific community in the 1970s. Nearly fifty years after the Apollo 17 mission these samples are being examined for the first time through the Apollo Next Generation Sample Analysis (ANGSA) Program. Given the precious nature of the Apollo 17 core samples, it is crucial to maximize scientific output before the samples are subjected to additional destructive investigations (e.g., bulk rock noble gas analyses). To that end, we have been allotted 17 small (between 2 and $16 \mathrm{mg}$ ) particles from various depths of the upper core 73002 for analysis through the ANGSA program for chemical analysis by electron probe microanalysis (EPMA) and noble gas analysis. Before these particles undergo melting for noble gas analyses, we utilized quantitative compositional stage mapping on the fragments as a way of maximizing the major- and minor-element chemical information from the sample, thus working to maximize the science return of the particles and providing critical context to noble gas analyses.

Lunar samples can present analytical challenges because they are often fine-grained and heavily brecciated owing to impact-processes on the Moon. Frequently, phases of interest are fine-grained clasts within a breccia matrix. Obtaining the bulk compositions of such clasts is analytically challenging, as bulk composition methods cannot isolate the clasts of interest, and electron probe microanalysis (EPMA) methods such as defocused beam analysis and modal recombination lack precision. However, quantitative compositional mapping has proved to be highly useful for the study of complex lunar samples [e.g., 1-4]. Quantitative compositional mapping, done by rastering the sample stage under a fixed electron beam, results in fully quantitative maps and chemical analyses for each pixel of the image. Thus, quantitative compositional mapping a much more complete analysis of major and minor elements in a sample than spot analysis or traditional X-ray intensity mapping alone.

Here we present quantitative compositional maps of several particles of 72003, along with spot analyses of individual minerals. Compositional data and back-scattered electron (BSE) images were collected using the JEOL 8530F electron microprobes at the Carnegie Institution for Science and the Smithsonian Museum of Natural History, each with 5 wavelength-dispersive spectrometers (WDS). Following the methods of [1], using JEOL and Probe for EPMA software, we collected maps of each particle of a varying size (up to $575 \times 530$ pixels) in order to maximize the area of the particle covered. Analytical conditions were $30 \mathrm{nA}$ probe current, with count times of 100 to $300 \mathrm{msec}$, step sizes of 1 to $2 \mu \mathrm{m}$, and spot sizes matching the step size. A standard WDS calibration was done prior to map collection and the WDS background removal was done by the mean atomic number (MAN) method. Typical acquisition time per sample was 15-30 hours for the three passes required to measure 14 common major and minor geologic elements. The result of this WDS stage mapping process is calibrated, $\Phi(\rho z)$ corrected (done iteratively 
in CalcImage software) images where each pixel contains a full WDS chemical analysis for the measured elements. The images were then visualized in Surfer (Golden Software) where chemical data for regions of interests in the sample is isolated and used to calculate the bulk composition of specific regions, e.g., clasts within a breccia. Additionally, the full chemical analysis taken at each pixel was filtered (e.g., for totals within $\sim 97-103 \%$ ) and output using the CalcImage software, which is used to examine mineral chemical trends.

2020011473002 Test_73002 186A Pass 1_00070_, Elemental Wt.\% 2020011473002 Test_73002 186A Pass 1_00070_, Elemental Wt.\% $\mathrm{Al} \mathrm{Wt} \%$
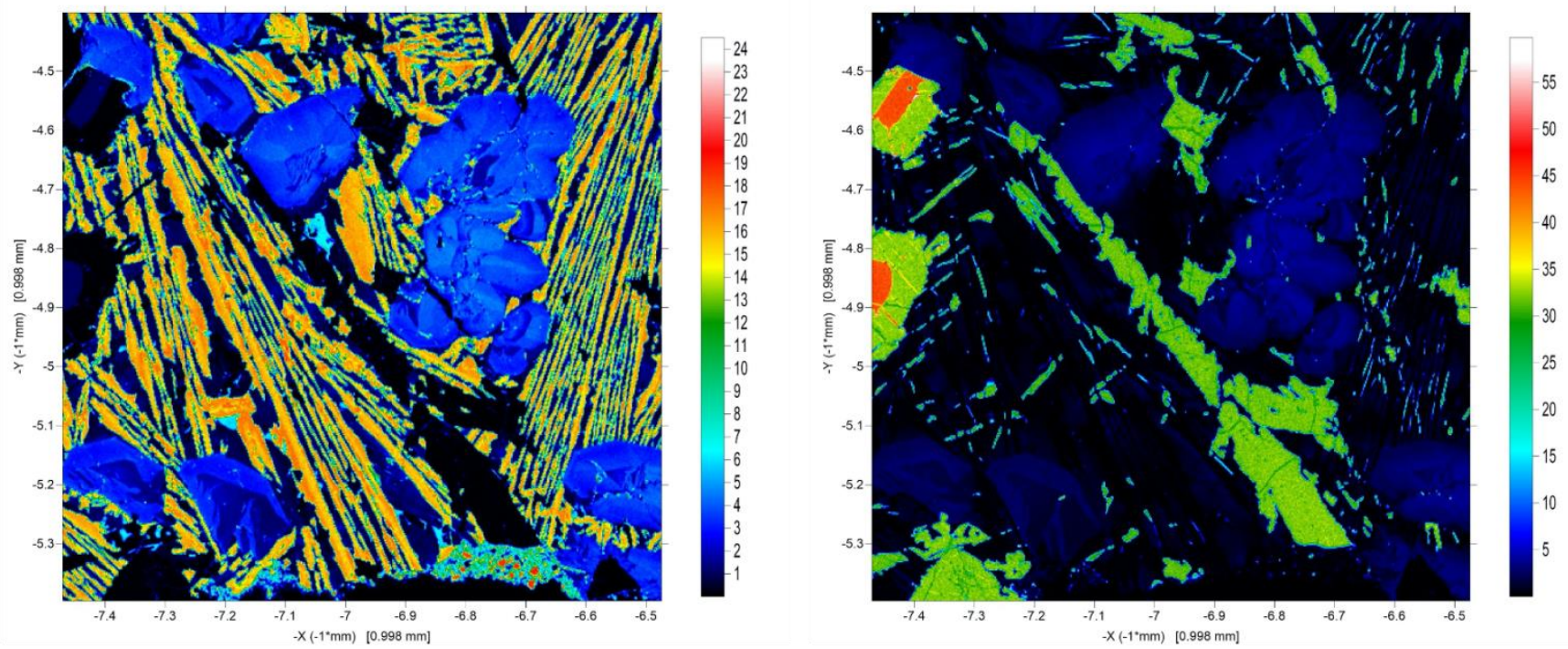

Figure 1. Example of quantitative compositional maps of a basaltic fragment collected from sample 73002. Each pixel in these maps corresponds to the intensity of $\mathrm{Al}$ (left) and $\mathrm{Ti}$ (right) characteristic $\mathrm{x}-$ rays and is fully quantitative.

\section{References}

[1] P C Carpenter et al, LPSC 44, Abstract \#1827 (2013).

[2] S N North-Valencia et al Microsc. Microanal. 20 (2014).

[3] P C Carpenter et al, LPSC 48, Abstract \#1964 (2017).

[4] T M Hahn et al Microsc. Microanal. 23 (2017). 